\title{
A Design of $\mathrm{PH}_{3}$ Monitoring System Based on NB-IoT
}

\author{
Zhu Tianpei ${ }^{a}$, Zang Chunhuab, Jin Yucheng ${ }^{c}$, Shi Xiang ${ }^{d}$ \\ College of Electronic and Information Engineering, Nanjing University of Aeronautics and Astronautics, 29 \\ Jiangjun Avenue, Nanjing, China \\ a zateper@foxmial.com, bzch403@nuaa.edu.cn, c530169320@qq.com, ${ }^{\mathrm{d}}$ 1466273754@qq.com
}

Keywords: NB-IoT, Phosphine, STM32, Low Power

Abstract: This paper describes a phosphine gas monitor based on NB IoT, which is designed by us and can be used to remotely measure the concentration of phosphine gas released during grain fumigation. The monitor uses the STM32 to control the sensor work, and after the measurement, upload the data to the network server through the NB IoT, and further data analysis can be performed on the server to achieve high accuracy, low power consumption (it can work for two years), low packet loss rate (less than $0.1 \%$ ) and so on.

\section{Introduction}

The use of phosphine in granaries is no residue, and the insecticidal rate is high, but the concentration range and fumigation time should be controlled. After releasing the drug, it is necessary to measure the concentration of the phosphine within a concentration range to ensure the insecticidal effect. Because the traditional need for manual sampling, the accuracy is low and unsafe for human. So, there are some wireless sensing methods such as ZigBee and other technologies, but it is easy to lose packets and other issues. [3] The NB IoT network has large coverage, stable signal, and low power consumption. Low loss packets, management automation as long as the battery-powered, easy to install the box, and the sensor measurement accuracy, also ensure personnel safety.

\section{Phosphine concentration measuring instrument}

\subsection{Overall Design.}

The overall design consists of three major parts: the measurement part, the controller part, and the communication part. The overall scheme architecture diagram is shown Fig. 1:

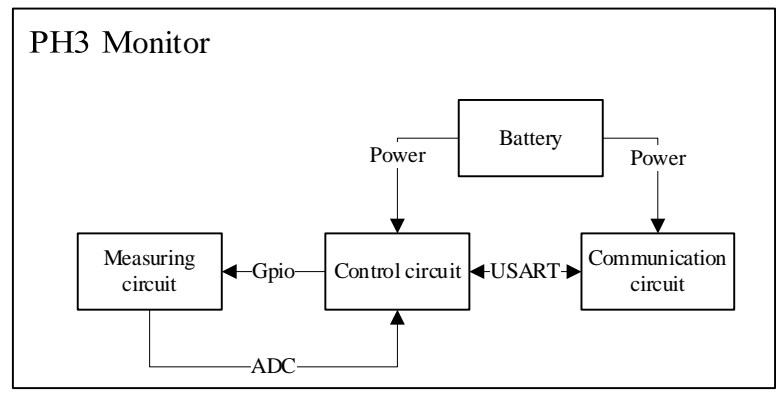

Fig. 1 overall scheme architecture diagram

The measurement part is an analog circuit designed to amplify the weak current signal from gas sensor and convert it to voltage signal so that MCU can collect gas concentration data through internal Analog-to-Digital Converter (ADC). It can measure the gas concentration range of 0 to $2000 \mathrm{ppm}$ of phosphine. The controller part is a minimum system of Microcontroller Unit(MCU), which is used to control the power-on of the measurement part and to collect the phosphine concentration data of the voltage measurement by the ADC. After the conversion, the digital value will be transmitted to the communication part. The communication part of the circuit mainly works 
with a NB-IoT module and is responsible for transparent transmission of data between the controller and the base station of telecom operator.

\subsection{Sensor Circuit Design.}

In order to measure phosphine concentration of large range, a Honeywell 7PH3-2000 0-2000ppm range sensor is used. It's sensing electrode (S), used to oxidize or reduce the gas, and produce a current proportional to the gas concentration. It has a linear relationship between the output signal and gas concentration on the recommended concentration range of the operating gas and can be calculated using the following formula:

output signal $(\mu \mathrm{A})=$ sensitivity $(\mu \mathrm{A} / \mathrm{ppm}) \times$ gas concentration $(\mathrm{ppm})$

The sensitivity is $0.07 \pm 0.04 \mu \mathrm{A} / \mathrm{ppm}$. We set the gain resistance as $10 \mathrm{k} \Omega$, and Vref is $0.5 \mathrm{~V}$, so:

$\mathrm{V}_{\text {ref }}=$ concentration $(\mathrm{ppm}) \times 0.07 \mu \mathrm{A} / \mathrm{ppm} \times 10 \mathrm{k} \Omega+0.5 \mathrm{~V}$

That is, the voltage step per ppm is $0.7 \mathrm{mV}$, and the concentration value is obtained:

Concentration $(\mathrm{ppm})=\left(\mathrm{V}_{\text {ref }}-0.5 \mathrm{~V}\right) \div 0.7(\mathrm{mV} / \mathrm{ppm})$

In this way, when the concentration is 2000, the voltage corresponding to the maximum measured concentration is $1.9 \mathrm{~V}$, then the voltage range corresponding to the concentration range is $0.5 \mathrm{~V}$ to $1.9 \mathrm{~V}$. Considering the maximum sensitivity of $0.11 \mu \mathrm{A} / \mathrm{ppm}$, the maximum Voltage value is $2.7 \mathrm{~V}$, so can inside the $\mathrm{ADC}$ measurement range from $0 \mathrm{~V}$ to $3 \mathrm{~V}$ that can meet the basic measurement range requirements.

\subsection{Controller Solution}

The control circuit of the phosphine concentration measuring instrument adopts the ST controller STM32L151 as the main controller. The core of the main control chip is ARM Cortex-M3 32-bit CPU, Ultra-low-power 32-bit MCU ARM-based Cortex-M3, 128KB Flash, 16KB SRAM, 4KB EEPROM [6].

The circuit composition of the minimum system of the MCU includes the power supply stability, external crystal oscillator and so on. The 8M passive crystal oscillator enables the MCU to be configured in the operating frequency of $8 \mathrm{MHz}$, at this time the operating power can be guaranteed below $5 \mathrm{~mA}$. After entering STOP mode, power consumption drops below $10 \mu \mathrm{A}$. We need to use RTC to wake up the chip from STOP mode. Since we can configure up to 40 seconds of wake-up time and actually need to work every 30 minutes, then we need to count every 10 second when we wake up from RTC. At 6×30=180 times, work once. Since the RTC interrupt can still enter and count while working, the working time does not affect the time interval.

\subsection{Communication Scheme}

\subsubsection{NB-IoT}

NB-IoT stands for Narrow Band Internet of Things(NB-IoT). It is built on a cellular network and consumes only about $180 \mathrm{kHz}$ of bandwidth. It can be directly deployed on GSM networks, UMTS networks, or LTE networks to reduce deployment costs and achieve smooth upgrades.

Internet of Things (IoT) devices can be classified into three categories. One type has a weak mobility but a large amount of data. A type has a small amount of mobile data, and a type has a weak mobility and a small amount of data. Narrowband Internet of Things belongs to the third category of Internet of Things. It has a small bandwidth and is not sensitive to delays, but it can transmit small amounts of data stably so that it can be used in environments with weak signals and large amounts of equipment. At the same time, it also brings features such as power consumption, minimum signaling overhead, and ultra-low cost.

\subsubsection{M5310}

M5310 is a small and easy-to-configure narrowband Internet of Things module produced by CMIoT (China Mobile IoT Company Limited). The internal HiSi Hi2110 supports China Mobile's 
network Band 8, which can operate between $3.1 \mathrm{~V}$ and $4.2 \mathrm{~V}$. In the PSM mode, the power consumption is less than $5 \mu \mathrm{A}$. There are two USART ports, one for application and the other for debugging.

\section{Measurement system software design}

\subsection{Software flow and low power design}

In the previous section on low-power design of hardware, the software also needed to match the hardware to meet the expected power requirements.

The official reference manual of STM32L151 describes that in the Run mode, different operating frequency power consumption is different, and the lower the frequency, the lower the power consumption $8 \mathrm{MHz}$, the corresponding current is $2.15 \mathrm{~mA}$, plus the current consumption of the pin to power the sensor circuit, basically meet the power consumption of $5 \mathrm{~mA}$.The following figure Fig. 2(a) describes the flow and overall system flow chart of the SCM switching different modes:

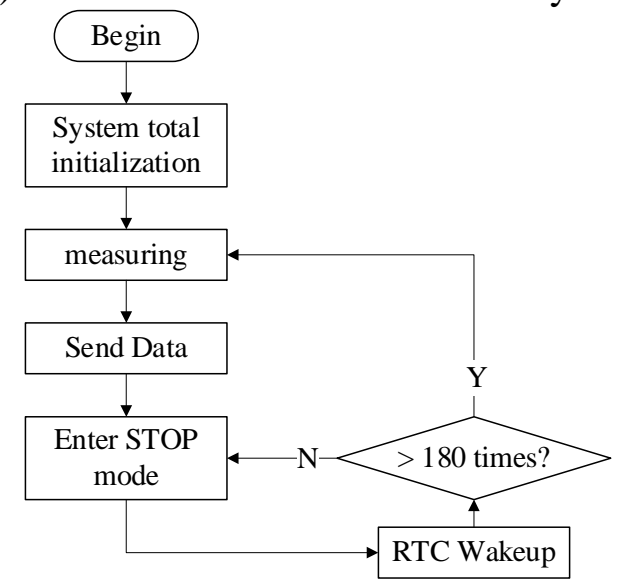

(a)

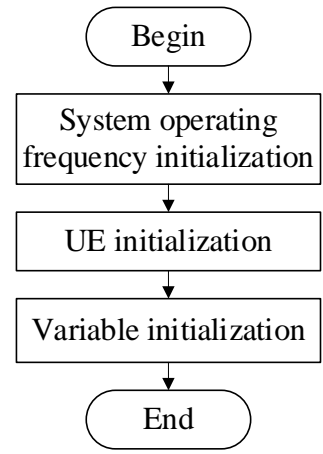

(b)

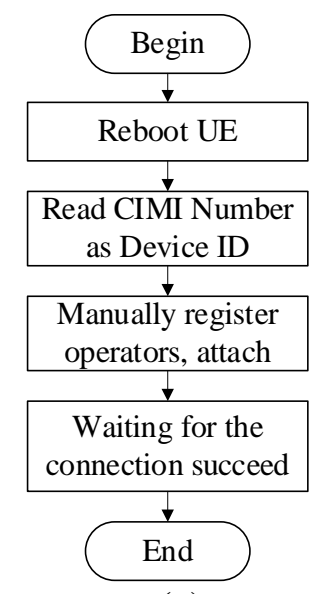

(c)

Fig. 2 Flows

\subsection{System initialization}

When the system is powered on, it needs to be initialized. The first step is to let the system work at the selected operating frequency. Then the module is ready and some parameter variables are initialized. These variables are used to indicate the number of acquisitions and the number of transmissions. The system initialization process is as shown Fig. 2(b).

UE initialization

Before configuring the module, use the reset pin to restart the module, so that the internal program of the module is initialized. When the module returns to OK, the CIMI number of the SIM is read and set as ID number, and it is determined that the SIM card is inserted and can be used. After restarting, the module will automatically look for the base station and the cell, and can manually request the module to register and attach to the operator's core network. A simplified flowchart of module initialization as shown Fig. 2(c).

\subsection{Measuring program}

The analog circuit is used to convert the current into voltage on the hardware, and the microcontroller is responsible for the ADC acquisition. To control the power-on and power-off of the conversion circuit, the IO pin of the microcontroller is directly used for the power supply during design. After reading the value, the pin can be pulled low to effect power off. After the voltage value is measured by the ADC, the program converts the voltage value to a concentration value using a formula. 


\subsection{Send Data}

After the measurement is completed, the data needs to be sent to the server immediately. The NB module supports UDP transmission. First, create a socket and associate with the specified protocol. After the socket is created, data can be sent to the server's fixed port through the UDP protocol. Since the information interaction protocol has been deployed on the server beforehand, as long as the data is sent in a fixed format, the server will automatically parse the data and save the valid information. Because UDP is not a very reliable transmission method, sending a request in advance is similar to a heartbeat. It is used to test whether the link with the server is unobstructed. After the server receives the heartbeat, it automatically returns the current timestamp, because the time difference before and after the difference is not large. Generally, phosphine gas will not change rapidly in the warehouse, so a few seconds of error is allowed.

After receiving the timestamp of the service, the timestamp can be packaged with the previous measurement value, ID number, etc., and then delivered to the module and sent by UDP to the port to which the server responds. After the server receives the data, it will return an Ack, which means that the server will receive data successfully. At this time, the microcontroller can exit from work and enter hibernation. If it can't receive the Ack, this time's data will be saved until the next response. So, every data will be sent to the server unless the number of data is full of memory, and then the earliest data will be deleted.

\section{System testing}

After the complete hardware circuit is soldered and programmed, the circuit board and battery are placed in a well-sealed box, and after the sensor is connected, the entire box is placed in a gas environment for measuring the concentration of phosphine. After the aluminum phosphide tablets are placed, the phosphine gas is slowly released. After a while, the gas concentration of the phosphine gas can be measured.

\subsection{Data reception test}

Since this chapter mainly describes the hardware and software implementation of the Monitor, the specific implementation of the server on the network is not described in detail. The basic flow is that when the first UDP data is received, the requested command is judged, and then the timestamp is received. The second UDP data is the measurement data, will be saved in SQL, and do analysis.

After connecting to the server's MySQL database through Navicat ${ }^{\circledR}$, you can see the data stored on the server, as shown in the following figure Fig. 3:

\begin{tabular}{|lllllll|}
\hline DEVICE_ID & & HZSPPM & ENERGY & COLLECT_TIME & import_time \\
\hline $0460 C$ & 08949 & 377.34 & 83.0 & $2018-04-08$ 09:52:04 & $2018-04-08$ 09:52:06 \\
\hline $0460 C$ & 08949 & 383.73 & 83.0 & $2018-04-0810: 03: 05$ & $2018-04-08$ 10:03:07 \\
\hline $0460 C$ & 08949 & 454.12 & 83.0 & $2018-04-0810: 14: 04$ & $2018-04-0810: 14: 06$ \\
\hline $0460 C$ & 08949 & 483.56 & 83.0 & $2018-04-0810: 25: 03$ & $2018-04-0810: 25: 05$ \\
\hline $0460 C$ & 08949 & 504.03 & 83.0 & $2018-04-0810: 36: 00$ & $2018-04-0810: 36: 02$ \\
\hline $0460 C$ & 08949 & 519.39 & 83.0 & $2018-04-0810: 46: 56$ & $2018-04-0810: 46: 58$ \\
\hline $0460 C$ & 08949 & 516.83 & 83.0 & $2018-04-0810: 57: 51$ & $2018-04-0810: 57: 53$ \\
\hline
\end{tabular}

Fig. 3 data received on the server

In the figure, HZSPPM is the measured phosphine gas concentration, Collect time is the time when the server receives the first UDP data, and import_time is the time when the server receives the second UDP data. Collect_time can be considered as the measurement time. Through calculations, it can be known that the two receiving intervals are basically within $2 \mathrm{~s}$, which means that using NB IoT network, the delay is basically about $1 \mathrm{~s}$, and one round trip requires $2 \mathrm{~s}$.

\subsection{Sensor conversion circuit linearity test}

In section 1.2, the datasheet of the sensor shows that the theoretical output value is linear, that is, the linear relationship between the concentration value and measured voltage value can be expressed as $y=k x+b$. In addition, when the conversion circuit is designed, it is also guaranteed that 
the conversion circuit is linear. In order to simply verify this, when the phosphine gas is released, the gas in the environment is sampled at intervals, and the calibration accuracy can also be verified. The sampling value is the abscissa, and the measured value is the ordinate. The measured points are plotted in the figure. The fitting curve and the goodness of fit $\mathrm{R}^{2}$ can be calculated by using Excel's own linear fitting. The plotted figure looks like Fig. 4:

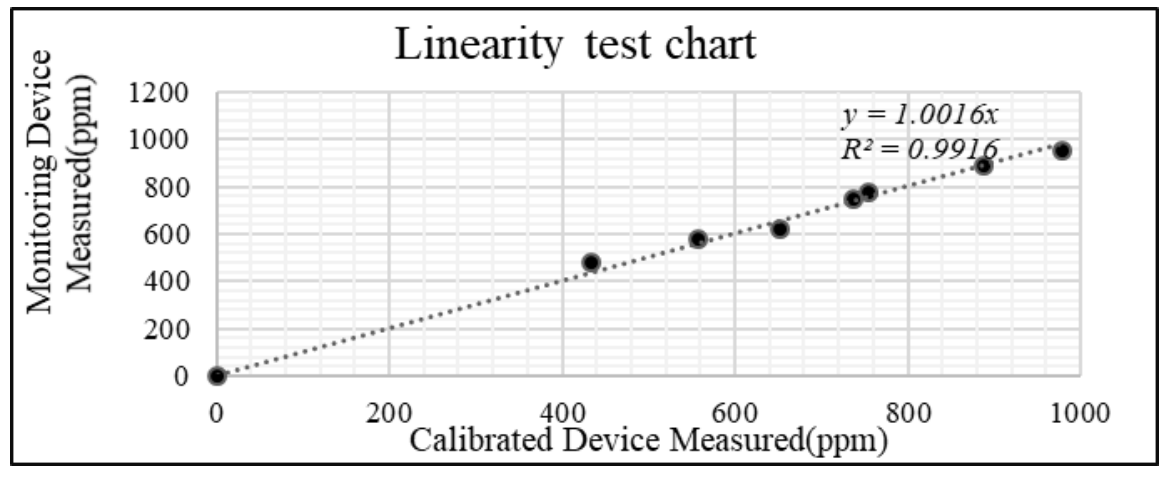

Fig. 4 Linearity test chart

1) It can be clearly seen that the measured values are very close to those of the existing measurement equipment, and the goodness of fit is above 0.99 , which can basically be tested to be indeed linear.

2) It can be seen from the chart that the basic error range is within $\pm 40 \mathrm{ppm}$, within the acceptable range of measurement accuracy.

\section{Conclusion}

The accuracy of phosphine detection by means of random sampling and other methods were low, with personnel security problems, and the workload was huge, and can't ensure that fumigation meets requirements. Now NB IoT network has the characteristics of stability, low power consumption and low packet loss rate. This design is not only the measurement is reliable and can be left unattended, easy to install, and the remote server automatically records the data. Compared with the judgement whether fumigation meets the requirements with the original manual experience, using this system to realize data visualization is more reliable and more scientific. Moreover, the large amount of historical data recorded can also provide a powerful data reference for improving the fumigation process in the future.

\section{References}

[1] Chaudhry M Q. Review A Review of the Mechanisms Involved in the Action of Phosphine as an Insecticide and Phosphine Resistance in Stored-Product Insects[J]. Pesticide Science, 1997, 49(49):213-228.

[2] Potdar V, Sharif A, Chang E. Wireless Sensor Networks: A Survey[J]. Computer Networks, 2002, 38(4):393-422.

[3] Wang Xiaodong, Lin Weiguo, Zhu Yuqi, et al. Toxic Gases Detection and Alarm System in Laboratory Based on ZigBee Wireless Sensing Network Technology[C]. //The 1st BUCT Students' Creativity Forum Proceeding, 2012:142-146.

[4] Beyene Y D, Jantti R, Ruttik K, et al. On the Performance of Narrow-Band Internet of Things (NB-IoT) [C]// Wireless Communications and NETWORKING Conference. IEEE, 2017:1-6.

[5] Ratasuk R, Vejlgaard B, Mangalvedhe N, et al. NB-IoT system for M2M communication[C]// Wireless Communications and NETWORKING Conference Workshops. IEEE, 2016:428-432.

[6] Information on http://www.st.com 